Title : will be set by the publisher

Editors : will be set by the publisher

EAS Publications Series, Vol. ?, 2021

\title{
FORBIDDEN CALCIUM LINES AS DISC TRACERS
}

\author{
Anna Aret $^{1}$ and Michaela Kraus ${ }^{1,2}$
}

\begin{abstract}
Forbidden emission lines are particularly valuable disc tracers, because their profiles reflect the kinematics within their formation region. Here we present a short excerpt from the results of a spectroscopic survey of evolved massive stars surrounded by high-density discs.
\end{abstract}

\section{Introduction}

Emission line stars are typically surrounded by large amount of dense circumstellar material (CSM) that often accumulates in rings or disc-like structures. Gas diagnostics using forbidden [O I] lines is well known: these lines arise in high-density environments, such as the inner disc regions around $\mathrm{B}[\mathrm{e}]$ supergiants. Recently we have discovered also [Ca II] $\lambda \lambda 7291,7324$ lines in the spectra of $\mathrm{B}[\mathrm{e}]$ supergiants, which trace hotter regions closer to the star than the [O I] lines (Aret et al. 2012). We initiated a survey of emission line stars in different evolutionary phases with various circumstellar environments to study physical conditions in the CSM.

Table 1. Stellar parameters and extracted disc kinematics

\begin{tabular}{lcll|lccc}
\hline \multirow{2}{*}{ Star } & $M_{*}$ & $i^{a}$ & Ref & \multicolumn{2}{|c}{$[\mathrm{Ca} \mathrm{II}]$} & \multicolumn{2}{c}{$[\mathrm{O} \mathrm{I}]$} \\
& {$\left[M_{\odot}\right]$} & $\left.{ }^{\circ}\right]$ & & {$[\mathrm{km} / \mathrm{s}]$} & \multicolumn{1}{c}{$[\mathrm{AU}]$} & $v_{\text {rot }}$ & $R$ \\
& {$[\mathrm{~km} / \mathrm{s}]$} & {$[\mathrm{AU}]$} \\
\hline V1478 Cyg & $38-40$ & 82 & $(1)$ & $38 \pm 1$ & $24.6 \pm 1.3$ & $25 \pm 1$ & $55.7 \pm 5.9$ \\
l Pup & $15-20$ & 38 & $(2)$ & $72 \pm 1$ & $3.0 \pm 0.4$ & $68 \pm 1$ & $3.4 \pm 0.5$ \\
V1429 Aql & $66 \pm 9$ & $73 \pm 13$ & $(3)$ & $50 \pm 1$ & $23.4 \pm 4.0$ & - & - \\
OY Gem & 0.62 & - & $(4)$ & 0 & - & 0 & - \\
\hline
\end{tabular}

${ }^{a}$ Disc inclination $i$ of $90^{\circ}$ means edge-on view of the disc.

(1) Báez-Rubio A., et al. 2013, A\&A, 553, A45; (2) Millour F., et al. 2011, A\&A, 526, A107; (3) Lobel A., et al. 2013, A\&A, 559, A16; (4) Arkhipova V. P., Ikonnikova N. P., 1992, Soviet Ast. Lett., 18, 418

\footnotetext{
1 Tartu Observatory, 61602, Tõravere, Tartumaa, Estonia

2 Astronomický ústav AV ČR, Fričova 298, 25165 Ondřejov, Czech Republic
} 
Title : will be set by the publisher

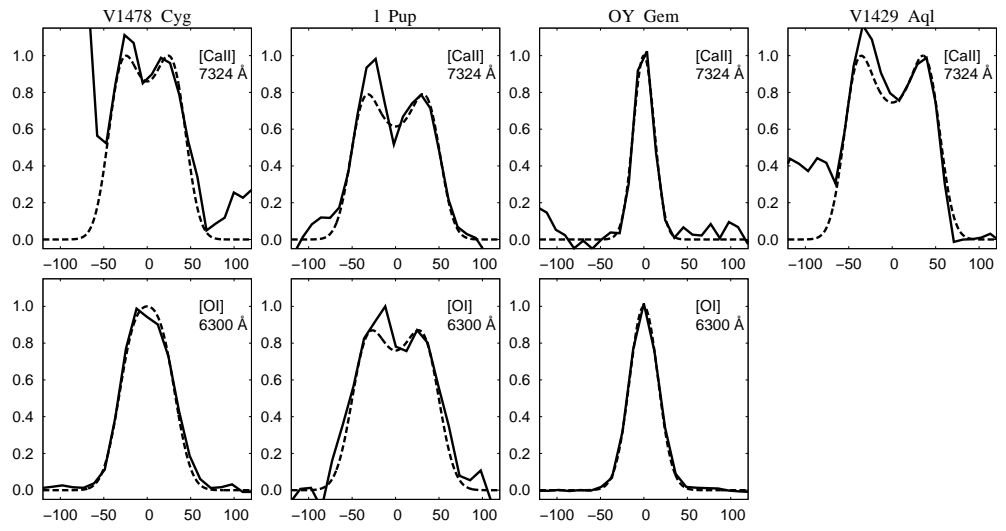

Fig. 1. Model fits (dashed) to the observed profiles (solid) of the forbidden lines.

\section{Observations and modelling}

The observations of the [O I] $\lambda 6300$ and the [Ca II] $\lambda \lambda 7291,7324$ lines were obtained using the Coudé spectrograph attached to the 2-m telescope at Ondřejov Observatory (Š́lechta and Škoda 2002) with a spectral resolution of $\sim 20 \mathrm{~km} \mathrm{~s}^{-1}$.

We applied a simple, purely kinematic model. Assuming the emission originates from a narrow Keplerian rotating ring, we calculated the profile shape considering only the rotational velocity, projected to the line of sight according to the observed inclination angles (Table 1), and the resolution of the spectrograph.

\section{Results}

The good fits to the observed line profiles (Figure 1) demonstrate that the observed emission originates indeed from a narrow ring region with radius $R$ (Table 1). For the two B[e] supergiants, V1478 Cyg and l Pup, the kinematics obtained from the $[\mathrm{O} \mathrm{I}]$ and $[\mathrm{Ca} \mathrm{II}]$ line profiles agrees with an origin of the lines in the Keplerian rotating disc. The LBV candidate V1429 Aql shows no [O I] lines, but the profile of its [Ca II] lines suggests that the emission originates in its hot, ionized circumbinary disc. The forbidden lines in the spectra of the compact planetary nebula OY Gem display no kinematical broadening beyond spectral resolution.

Acknowledgements: A.A. acknowledges financial support from Estonian grants ETF8906 and IUT40-1; M.K. from GA ČR (14-21373S) and RVO:67985815.

\section{References}

Aret, A., Kraus, M., Muratore, M. F., and Borges Fernandes, M.: 2012, MNRAS, 423, 284

Šlechta, M. and Škoda, P.: 2002, Publ. Astron. Inst. Acad. Sci. Czech Republic, 90, 1 